\section{Tiempos de crisis: la brecha como oportunidad de diseño}

Ervin Manuel Moreno Velásquez ${ }^{(1)}$ y Kevinn Rodrigo Mayén López ${ }^{(2)}$

Resumen: Cuando la sociedad se ve interrumpida por alguna adversidad, las personas tienen que adaptarse al nuevo estilo de vida de manera indefinida. El cambio ocurre cuando los sucesos salen de las manos y se es forzado a evolucionar para satisfacer las necesidades. En las situaciones de emergencia es cuando la sociedad es obligada a adaptarse, en este punto es como un diseñador satisface la nueva necesidad o abre una nueva brecha para ser explorada. El diseño empieza por una necesidad vista como una ventana para mejorar el estilo de vida de la sociedad, las emergencias impulsan a crear o mejorar soluciones de una manera eficaz. El objetivo de este estudio es determinar las acciones que se tomaron ante la pandemia COVID-19, centralizada en el contexto guatemalteco.

Palabras clave: Diseño Industrial - prototipo - tecnología alternativa - innovación - emergencia.

[Resúmenes en inglés y portugués en la página 182]

(1) Diseñador Industrial (Universidad Rafael Landívar). Coordinador del Centro de investigación, capacitación y diseño en tecnologías alternativas (CTA) para el Instituto de investigación y estudios superiores en arquitectura y diseño (Indis).

(2) Becado de Arte y Cultura (Universidad Rafael Landívar), Estudiante Diseño Industrial. Coordinador del Centro de Investigación, Capacitación Y Diseño en Tecnologías Alternativas - CTA - Subprograma del Instituto de Investigación en Estudios Superiores de Arquitectura y Diseño (INDIS).

\title{
Introducción
}

El diseño es una herramienta de evolución constante, por lo que va ligado a los cambios socioeconómicos que enfrenta un país o el mundo entero, brindando soluciones rápidas y puntuales. Como en las epidemias que han ocurrido en tiempos anteriores, la economía es afectada y los suministros que son requeridos para prevención y cura son escasos por lo que la demanda sube y la capacidad de producción es escasa. 
En medio de la pandemia del covid-19 o SARS-CoV-2 se está viviendo algo que muchos llaman la cuarta revolución industrial ya que los procesos de producción de una empresa están altamente automatizados y controlados digitalmente, así mismo la investigación de su grupo objetivo es más preciso con ayuda de la interacción de las personas con sus teléfonos móviles y las redes sociales alimentando algoritmos personalizados de acuerdo a nuestras necesidades y deseos de compra, esperando que esta información recopilada sea moralmente manejada. La información es almacenada en la nube, por lo que permite el acceso de trabajar remotamente ya que todo lo necesario se encuentra almacenado. Puede ser que no todo el mundo esté viviendo la cuarta revolución industrial, sin embargo, la tecnología desarrollada se vuelve más accesible, uso de redes sociales, almacenamiento en la nube, o maquinaria de fabricación digital.

\section{La impresión 3D}

Este proceso de producción está clasificado dentro de la fabricación digital que puede definirse como "la digitalización de los procesos de fabricación" (Ortega, 2016, p. 5). Este tipo de herramientas tienen una función lo más cercano a ser autónomas, pudiendo replicar piezas exactas una tras otra o poder personalizar cada pieza que produzca. Estas máquinas evolucionan de tener sistemas integrados, como las herramientas que conocemos como un microondas, por ejemplo, a un sistema automatizado CNC (Computer Numeric Control). Como toda herramienta de fabricación digital, necesita información u órdenes para poder trabajar, por lo que requiere de un modelo tridimensional digital, un software CAD (Computer Aided Design) y un software que interprete el modelo digital en códigos de programación para que la máquina funcione. Ahora bien, una máquina de fabricación digital puede funcionar de manera autónoma toda vez que se tenga la materia prima y se de la orden de realizar el trabajo, esto funciona como el microondas del ejemplo anterior, con una combinación de comandos para hacerlo funcionar. Con la cuarta revolución industrial una herramienta de fabricación digital puede ser controlada de manera remota, conectada a una red y poder configurar simultáneamente diferentes herramientas.

La impresión 3D es conocida como un proceso de manufactura aditiva, esto significa que un objeto lo genera agregando material y no sustrayendo, como lo es en la escultura. La aplicación de esta herramienta es muy amplia, existen impresoras 3D capaces de generar piezas de metal, vidrio, plástico, inclusive materiales cementantes. Si una impresión 3D agrega material, ¿cómo se genera la pieza? A través de coordenadas computarizadas indica dónde debe depositar el material y en cuánta cantidad. Una impresión 3D se genera de manera vertical cortando, virtualmente, el objeto y luego esos cortes se van apilando uno sobre otro la forma deseada del objeto.

Características de la impresión 3D: reduce tiempos de producción, pueden realizarse pequeñas producciones industriales, personalización de diseños (diseño a la medida), materialización de objetos que serían imposibles de replicar en cualquier producción tradicional. Existen diferentes gamas de impresión 3D, impresoras que pueden llegar a costar miles de dólares ya que utilizan tecnologías innovadoras que están todavía en desarrollo y ma- 
teriales poco accesibles para cualquier persona. Hay impresoras de escritorio que pueden costar cien dólares, que funcionen adecuadamente.

El mayor beneficio que brinda la impresión 3D, independientemente de la gama y tipo de impresora, es cuando una idea se convierte en un objeto tangible en cuestión de horas, permitiendo realizar iteraciones de un diseño con mayor facilidad.

\section{El caso de protectores faciales impresos en 3D}

Resultado de la pandemia, los médicos y personal involucrado en los hospitales o establecimientos que combaten el covid-19 se vieron en la necesidad de uso equipo de protección personal (EPP), este equipo está diseñado para proteger las zonas más vulnerables de la persona que está haciendo el uso de esta, protegiendo áreas como la boca, la nariz, los ojos y la piel. Evidentemente, necesitan el uso de EPP con mayor razón porque se exponen a personas contagiadas realizando su honorable labor. El EPP adecuado consiste en: uso de mascarilla n95, gafas o protector facial, gorro, bata, guantes y zapatones.

Existen empresas dedicadas a la fabricación de estos equipos de manera industrial. Sin embargo, de acuerdo con la necesidad mundial actual es escaso por la alta demanda.

¿Por qué un protector facial? Un protector facial previene que el virus entre en contacto con los ojos y región de la cara. También es un complemento del uso de una mascarilla ya que los puntos más probables de contagio se encuentran en la cara, inclusive por un accidente propio al momento de pasar alguna extremidad cerca de los ojos o la cara. Cuando se realiza la prueba del hisopado, el paciente puede toser, estornudar o inclusive vomitar, por lo que es crucial el uso de un protector facial. Es posible que se haya elegido un protector facial para fabricar no solo por la demanda sino por el material de fabricación, siendo más accesible para la fabricación.

Un protector facial consiste principalmente de tres piezas: visor, pieza de plástico transparente flexible como PP, Acetato o PET; diadema, es la estructura que sostiene el visor, usualmente plástico, en este caso para impresión 3D, PLA o PETG; y una banda elástica, cuya función es evitar que el protector facial se caiga.

Una empresa dedicada a la producción de impresoras 3D tuvo la iniciativa de producir protectores faciales. Prusa Research, fundada en el año 2010 en Republica Checa, es relativamente nueva en el mercado de impresión 3D. Su fundador Josef Prusa empezó a adentrarse en el mundo de la impresión 3D como un pasatiempo para realizar prototipos personales relacionados con la música electrónica, con el pasar del tiempo se enfocó en mejorar el diseño de impresoras de código abierto, modificando el software y el hardware, liberando los diseños para que cualquiera pueda usarlos. Con el pasar del tiempo, desarrolló una impresora con nuevas piezas y las empezó a comercializar, vendiendo un par de impresoras al mes. Muchas de las piezas las imprimía él mismo. Actualmente tienen 500 impresoras 3D dedicadas específicamente para la producción de partes para las máquinas que vende. Así mismo, producen piezas que les facilita la línea de ensamblado y testeo de las mismas. 
Prusa Research desarrolló protectores faciales impresos en 3D de acuerdo con las necesidades y capacidades de producción de la empresa. Todo surgió en un grupo en la red social de Facebook llamado "Covid19CZ" en el cual Prusa estaba al tanto. Las solicitudes de protectores faciales era lo que resaltaba en el grupo, ese mismo día en Prusa Research desarrollaron un prototipo, impreso en 3D, para validar su uso, luego crearon una línea de producción enfocada en los protectores faciales. Desarrollaron instructivos de cómo imprimir los protectores y qué materiales usar. Adaptaron su fábrica de impresoras 3D en una fábrica de protectores faciales, todo esto en tres días. Su capacidad de producción al día era de 3,000 protectores faciales. La aceptación de los protectores fue positiva y de gran ayuda para los hospitales de su país. Con la tecnología de impresión 3D a la mano, mejoraron los procesos de producción, por ejemplo, ellos necesitaban cortar sus bandas elásticas y en un día desarrollaron una máquina específica para esa labor. También crearon una estación de desinfección de sus productos en poco tiempo. Actualmente, Prusa Research es capaz de producir 10,000 protectores faciales al día y en menos de un mes alcanzaron a producir más de 100.000. El diseño del modelo fue lanzado al mundo como código abierto, pudiendo ser descargado e impreso por personas de todo el mundo. Se obtuvieron más de 250.000 descargas de los archivos en menos de tres semanas, por lo que, en casi todas partes del mundo del ámbito de la impresión 3D, se enteraron de la hazaña que Prusa Research estaba realizando.

Este movimiento impulsó a que, cualquier persona con acceso a una impresora 3D pueda producir estos protectores faciales y adecuarlos a la necesidad y capacidad de cada uno. 3D Crowd UK, una comunidad de impresión 3D que surgió por la pandemia, produjo más 65,000 protectores faciales en cuatro semanas, con más de 8,000 voluntarios que se reparten los roles de impresión 3D, gestión de empaque y transporte de los protectores a diferentes hospitales del Reino Unido.

En Guatemala, así como en otros países, se creó una comunidad de makers llamada MakersGT. Esta comunidad surgió en las redes sociales en un grupo de interés común, siendo este, la impresión 3D. La comunidad denominada MakersGT fue creada por la iniciativa principalmente por dos personas: Jeffri Salazar y Kurt Kellner. Ellos, en conjunto con entusiastas y profesionales de la impresión 3D, ingenieros, diseñadores y personas con experiencia en la fabricación digital, plantearon replicar la iniciativa de Prusa Research. Se discutió sobre seguir el diseño de los protectores faciales o rediseñar la estructura del protector, se tomó en cuenta que Guatemala no es un país productor de material especializado en impresión 3D y la materia prima era la que se tenía a disposición de cada voluntario ya que era muy complicado de conseguir, especialmente por los retrasos de importación. En la comunidad se realizó la primera iteración del diseño, beneficiando el tiempo de impresión 3D y el peso de la impresión. El Centro de investigación, capacitación y diseño en tecnologías alternativas (CTA), realizó un diseño que se puede imprimir en 28 minutos pesando 13 gramos. Comparándolo con el modelo de Prusa Research que el peso es de 40 gramos aproximadamente y puede llegar a imprimirse en 1 hora y 20 minutos. El propósito de la modificación es aprovechar al máximo el filamento disponible, que es muy limitado.

En el CTA se imprimieron 700 protectores faciales en el transcurso de dos semanas. Se usaron diez impresoras 3D. Los retos que se enfrentó al realizar los protectores faciales fue 
la obtención de materia prima ya que el filamento PLA estaba escaso en Guatemala. Así mismo, el material del visor en acetato era escaso. También, de acuerdo a las disposiciones gubernamentales, estaba restringido el horario de la locomoción y los comercios con tiendas físicas estaban cerrados. Por lo tanto, conseguir materia prima significaba un reto adicional. El CTA que trabajó en conjunto con MakersGT, pudo obtener un recuento a nivel nacional de más de 200 impresoras 3D y más de 2,500 protectores faciales donados en menos de tres semanas.

La masificación de protectores faciales es un claro ejemplo de innovación en producción y de velocidad de respuesta ante las necesidades que requiere la sociedad en cuanto productos. Toda persona, empresa, proceso o producto tiene que estar en constante innovación y estar preparado para cualquier adversidad. Los protectores faciales es uno de muchos ejemplos que se vivió para esta pandemia de manera mundial y se puede aprender mucho de esto. Por ejemplo, el uso de fabricación digital para prototipado y producto final, el manejo de comunicación de manera virtual, esta herramienta ha existido por años y ha sido poco usada, hasta que se vio forzado a migrar las reuniones de trabajo, la modalidad de almacenamiento de datos en la nube. Estas herramientas resultan ser una opción con gran potencial para poder seguir evolucionando. También se puede implementar la producción con fabricación digital, donde una línea de producción puede mutar fácilmente de acuerdo con las exigencias que requieran en ese momento.

Hay que resguardarse para disminuir el pico de infecciones. Sin embargo, en un futuro cercano, inevitablemente se tendrá que seguir con las labores habituales como trabajar, estudiar o inclusive a realizar actividades recreativas; ya que muchas personas y empresas dependen económicamente de ello también. Esto abre un campo nuevo para poder innovar en la experiencia del día a día, como diseñadores, es una oportunidad para poder satisfacer las nuevas necesidades, para poder seguir socializando y a la vez estar protegidos.

\section{Listado de referencias bibliográficas}

Jorquera Ortega, A. (2016). Fabricación digital: Introducción al modelado e impresión 3D. España: Ministerio de Educación, Cultura y Deporte.

Schwab, K. (2016). La cuarta revolución industrial. Debate.

Tomasi, S. N. (2020). Historia de las pandemias mundiales y la economía.

Medical Devices and the COVID-19 Pandemic (6 de marzo de 2020). Recuperado de https:// www.fda.gov/medical-devices/emergency-situations-medical-devices/medical-devicesand-covid-19-pandemic\#PPE

3D PRINTED FACE SHIELDS FOR MEDICS AND PROFESSIONALS, (s.f). Recuperado de https://www.prusa3d.com/covid19/\#_ga=2.106378113.361685737.15910399691220415571.1586838691

WE ARE PRINTING TOGETHER TO SAVE LIVES, (s.f). Recuperado de https://3dcrowd. org.uk/about-us/

[Prusa Face Shield - US version] (1 de abril de 2020). Recuperado de https://www.prusa printers.org/prints/27950-prusa-face-shield-us-version 
[Prusa 3D by Josef Prusa]. (2020, junio 28). The Road to 100,000 Original Prusa 3D printers [Archivo de video]. Recuperado de https://www.youtube.com/watch?v=xX3pDDi9PeU

\begin{abstract}
When society is interrupted by some adversity, people have to adapt to the new lifestyle indefinitely. Change occurs when events get out of hand and you are forced to evolve to meet needs. In emergency situations it is when society is forced to adapt, at this point it is how a designer satisfies the new need or opens a new gap to be explored. Design begins with a need seen as a window to improve the lifestyle of society, emergencies drive to create or improve solutions in an effective way. The objective of this study is to determine the actions that were taken in the face of the COVID-19 pandemic, centralized in the Guatemalan context.
\end{abstract}

Keywords: Industrial Design - prototype - alternative technology - innovation - emergency.

Resumo: Quando a sociedade é interrompida por alguma adversidade, as pessoas precisam se adaptar indefinidamente ao novo estilo de vida. A mudança ocorre quando os eventos ficam fora de controle e você é forçado a evoluir para atender às necessidades. Em situações de emergência, é quando a sociedade é forçada a se adaptar, nesse ponto é como um projetista satisfaz a nova necessidade ou abre uma nova lacuna a ser explorada. $\mathrm{O}$ design começa com uma necessidade vista como uma janela para melhorar o estilo de vida da sociedade; as emergências buscam criar ou melhorar soluções de maneira eficaz. O objetivo deste estudo é determinar as ações que foram tomadas diante da pandemia do COVID-19, centralizada no contexto guatemalteco.

Palavras chave: Desenho Industrial - protótipo - tecnologia alternativa - inovação - emergência.

[Las traducciones de los abstracts fueron supervisadas por el autor de cada artículo] 